\title{
Effect of past and concurrent body mass index on prevalence of glucose intolerance and Type 2 (non-insulin-dependent) diabetes and on insulin response
}

\author{
The Israel study of glucose intolerance, obesity and hypertension
}

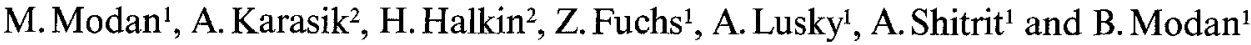 \\ Departments of Clinical Epidemiology ${ }^{1}$ and Medicine ${ }^{2}$, Chaim Sheba Medical Center, Tel Hashomer, \\ affiliated with the Tel Aviv University Sackler School of Medicine, Israel
}

\begin{abstract}
Summary. A representative sample $(n=2140)$ of the Israeli Jewish population aged 40-70 (excluding known diabetic patients), whose body mass index had been measured 10 years earlier, underwent an oral glucose tolerance test and redetermination of body mass index. Irrespective of weight changes, high concurrent and high past body mass index values $(\geq 27)$ were associated with similarly increased rates of glucose intolerance as compared with body mass index values $<27$ at both time-points (rate ratio $1.76,90 \%$ confidence limits $1.56-1.99$ ). Glucose intolerance here includes borderline and impaired tolerance as well as Type 2 diabetes. The rate of Type 2 diabetes increased only with increasing past body mass index, while concurrent body mass index had no effect [rate ratios: 2.36 $(1.48-3.75)$ and $1.99(1.48-2.68)$ respectively for the medium(23-26.9) versus-low $(<23)$ and high- $(\geq 27)$ versus-medium past body-mass-index categories]. Weight reduction was associated with only slightly reduced rate of glucose intolerance and had no effect on the rate of diabetes. Mean sum insulin (summed 1 and $2 \mathrm{~h}$ levels, $\mathrm{mU} / \mathrm{l}$ ) increased significantly with increasing concurrent body mass index $(123,150$ and 190 in
\end{abstract}

the low, medium and high categories) with no effect of past body mass index. It also increased significantly $(p<0.001)$ in all concurrent body mass index categories from normal tolerance through borderline to impaired tolerance, and decreased significantly $(p<0.001)$ in diabetes relative to impaired tolerance, although it remained above normal. Means of sum insulin within each glucose tolerance level were similar in the two lower concurrent body mass index categories, with markedly higher $(p<0.001)$ levels in the high body mass index category. All these findings held after accounting for age, sex, ethnic group and use of antihypertensive medications. We conclude that body mass index $\geq 27$ leads to early impairment in glucose tolerance. A prolonged period of obesity is apparently required for the development of Type 2 diabetes and its associated reduced insulin response. The reversibility of the deterioration of glucose tolerance seems to be limited.

Key words: Prevalence of Type 2 diabetes, prevalence of glucose intolerance, past and concurrent overweight, insulin response, hyperinsulinaemia.
Obesity has long been implicated in the aetiology of Type 2 (non-insulin-dependent) diabetes mellitus and impaired glucose tolerance [1-6]. All these conditions are characterized by hyperinsulinaemia reflecting insulin resistance [7-14]. Short-term follow-up studies of small selected groups have shown improvement of glucose tolerance, hyperinsulinaemia and insulin resistance consequent on weight reduction, and their worsening following weight gain [15-16]. Association of Type 2 diabetes and impaired glucose tolerance with concurrent obesity has been demonstrated by numerous large-scale cross-sectional epidemiological studies [1-3, $5,6]$. Association of past degree of obesity with subsequent development of Type 2 diabetes has been shown by a number of longitudinal studies [17-20], but the relative impacts of past and concurrent obesity were not assessed and no reference was made to impaired glucose tolerance. None of these studies addressed the possibility that the effect of overweight could be due to the increased burden of antihypertensive medications asso- ciated with the higher rate of hypertension in obese subjects, as these medications in themselves lead to deterioration of glucose tolerance [21, 22]. To the best of our knowledge, no population study has examined the effect of past obesity on insulin response.

The current report on a representative sample of the adult Jewish population in Israel evaluates the interplay of the effects of past and concurrent degree of obesity on the prevalence of the full range of glucose intolerance categories as well as on insulin response. Allowance is made for use of antihypertensive medications.

\section{Subjects and methods}

\footnotetext{
The study is based on a subgroup of the Israel Study of Glucose Intolerance, Obesity and Hypertension (The Israel GOH Study) a continuing nationwide longitudinal study (started in 1969) of a sample of 5711 individuals drawn from the Israel Central Population Registry and born between 1912 and 1941. Between 1969 and 1972 (Phase I) all participants were interviewed in their homes for regular use of medi-
} 


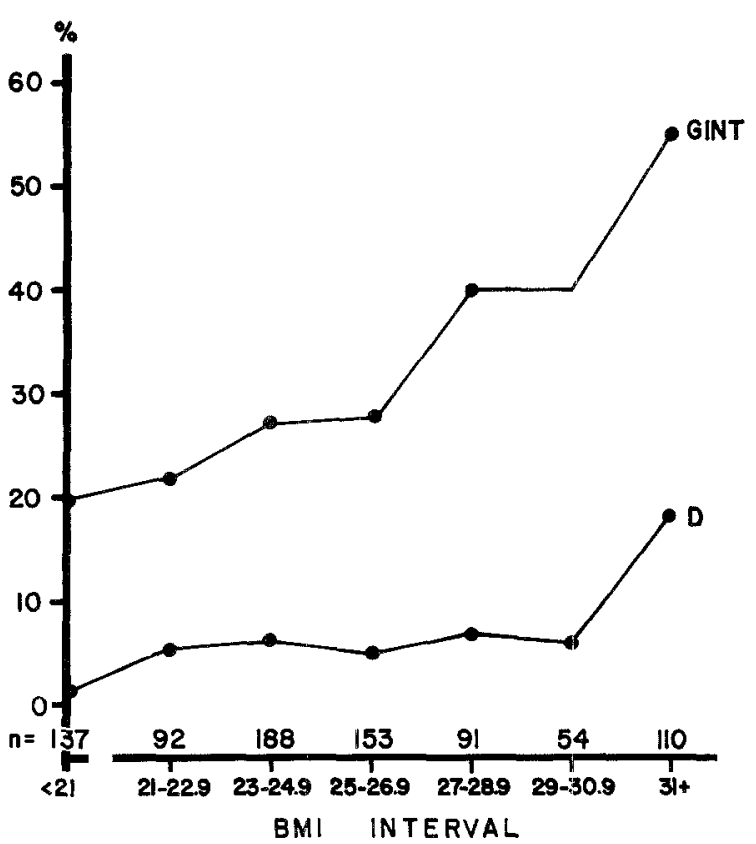

Fig. 1. Prevalence (\%) of glucose intolerance (GINT) and Type 2 diabetes (D) by body mass index (BMI) in the 825 individuals whose concurrent and past BMI were in the same two-unit intervals

cations. In additions, their weight, height and blood pressure were measured. Between 1977 and 1982 (Phase II), 2769 participants, a representative subgroup resembling the original sample in distributions of age, sex, ethnic group, body mass index (BMI) and blood pressure [23], attended regional medical centres after having the same variables recorded at home, where four blood pressure measurements were obtained - two before and two after the interview. The detailed sampling procedure and design in both phases have been reported elsewhere [23]. At the centre, participants not known to be diabetic were asked to undergo an oral glucose tolerance test (OGTT). From 1979 to 1982 , determination of insulin response to the oral glucose load was added to the protocol and determined in the most recent 1200 participants [24]. The current report addresses results from 2140 participants who underwent a full OGTT and whose weight and height were recorded in both phases with a mean interval of about 10 years. (The remaining cases comprise 191 previously-known diabetic patients, 294 OGTT refusals or technical problems, and 144 with incomplete data on weight or height). In a consecutive series of 362 individuals examined by OGTT at the beginning of Phase II and not diabetic, glucose tolerance and weight were determined again following a mean interval of 3.5 years. A standardized, detailed, quantitative dietary history, covering a year-round profile as well a 24 -h recall, was obtained for 632 randomly-selected individuals in whom insulin was measured. Both subsamples resembled the group of 2140 individuals in terms of age, sex, ethnic origin, BMI and blood pressure. No specific dietary instructions were given for the period preceding the OGTT, since compliance could not be ascertained. However, the dietary interviews indicated a daily intake of at least $250 \mathrm{~g}$ carbohydrate in the majority of participants, and the proportion of subjects with carbohydrate intake $<250 \mathrm{~g} /$ day was similar in all glucose tolerance categories (unpublished data); only a daily intake under $150 \mathrm{~g}$ affects test results appreciably [6]. Blood samples were drawn after an overnight fast and 1 and $2 \mathrm{~h}$ after a $100-\mathrm{g}$ oral glucose load. While the recommended load for determination of glucose tolerance level is $75 \mathrm{~g}$, the difference between these two loads is known to have little influence on blood glucose levels [6]. The 100-g oral glucose load was selected for its greater stimulation of insulin secretion [25].

Plasma glucose was determined by routine automated Technicon Autoanalyser II based on ferricyanide reduction. Plasma insulin $(\mathrm{mU} / \mathrm{l})$ was determined in duplicate by Phadebas Radioimmuno-
Table 1. Distribution of 2140 participants by changes in BMI category over 10 years

\begin{tabular}{llll}
\hline & \multicolumn{2}{l}{ Past BMI category } \\
\cline { 2 - 3 } & $\begin{array}{l}\text { Higher than } \\
\text { concurrent }\end{array}$ & No change & $\begin{array}{l}\text { Lower than } \\
\text { concurrent }\end{array}$ \\
\hline Concurrent BMI: & & & \\
Low $(<23)$ & $92^{\mathrm{a}}$ & 356 & $\mathrm{X}$ \\
Medium $(23-26.9)$ & 90 & 566 & 269 \\
High $(27+)$ & $\mathrm{X}$ & 470 & $297^{\mathrm{b}}$ \\
\hline
\end{tabular}

a In 87 of these individuals, past BMI was in the medium, and in 5, in the high category

${ }^{b}$ In 267 of these individuals, past BMI was in the medium, and in 30 , in the low category

$\mathrm{X}$ A non-existent category, by definition

Table 2. Prevalence of glucose intolerance (GINT) by BMI category, sex, age, ethnic group and blood pressure category in the no change group

\begin{tabular}{|c|c|c|c|c|c|c|}
\hline & \multicolumn{6}{|c|}{ BMI category } \\
\hline & \multicolumn{2}{|l|}{ Low } & \multicolumn{2}{|c|}{ Medium } & \multicolumn{2}{|c|}{ High } \\
\hline & No. & $\%$ GINT & No. & $\%$ GINT & No. & $\%$ GINT \\
\hline Total & 356 & 22.4 & 566 & 27.4 & 470 & 47.0 \\
\hline $\begin{array}{l}\text { Sex } \\
\text { Males } \\
\text { Females }\end{array}$ & $\begin{array}{l}171 \\
185\end{array}$ & $\begin{array}{l}25.7 \\
19.5\end{array}$ & $\begin{array}{l}344 \\
222\end{array}$ & $\begin{array}{l}33.7 \\
17.6\end{array}$ & $\begin{array}{l}218 \\
252\end{array}$ & $\begin{array}{l}48.2 \\
46.0\end{array}$ \\
\hline $\begin{array}{l}\text { Age } \\
40-49 \\
50-59 \\
60-69\end{array}$ & $\begin{array}{r}159 \\
112 \\
85\end{array}$ & $\begin{array}{l}13.2 \\
24.1 \\
37.6\end{array}$ & $\begin{array}{l}194 \\
218 \\
154\end{array}$ & $\begin{array}{l}16.0 \\
27.5 \\
41.6\end{array}$ & $\begin{array}{l}124 \\
194 \\
152\end{array}$ & $\begin{array}{l}35.5 \\
46.4 \\
57.2\end{array}$ \\
\hline $\begin{array}{l}\text { Ethnic group } \\
\text { Yemenite } \\
\text { Mid Eastern } \\
\text { North African } \\
\text { European }\end{array}$ & $\begin{array}{r}125 \\
85 \\
45 \\
101\end{array}$ & $\begin{array}{l}20.0 \\
29.4 \\
26.7 \\
17.8\end{array}$ & $\begin{array}{r}112 \\
137 \\
97 \\
220\end{array}$ & $\begin{array}{l}38.4 \\
23.4 \\
25.8 \\
25.0\end{array}$ & $\begin{array}{r}69 \\
122 \\
133 \\
146\end{array}$ & $\begin{array}{l}59.4 \\
40.2 \\
44.4 \\
49.3\end{array}$ \\
\hline $\begin{array}{l}\text { Blood pressure } \\
\text { category }\end{array}$ & & & & & & \\
\hline $\begin{array}{l}\text { Normotensive } \\
\text { Untreated } \\
\text { Hypertensive }\end{array}$ & $\begin{array}{r}286 \\
44\end{array}$ & $\begin{array}{l}18.9 \\
34.1\end{array}$ & $\begin{array}{l}364 \\
120\end{array}$ & $\begin{array}{l}21.1 \\
36.7\end{array}$ & $\begin{array}{l}214 \\
144\end{array}$ & $\begin{array}{l}40.7 \\
45.8\end{array}$ \\
\hline $\begin{array}{l}\text { Treated } \\
\text { Hypertensive }\end{array}$ & 26 & 38.5 & 82 & 41.5 & 112 & 61.6 \\
\hline
\end{tabular}

assay Kit (Pharmacia Diagnostics), the coefficients of variation being $4 \%$ within assay and $8 \%$ between assay.

Body mass index (BMI - weight in $\mathrm{kg} /(\text { height in } \mathrm{m})^{2}$ ) was used to express relative weight. Past BMI refers to Phase I. Concurrent BMI refers to that at the time of the OGTT in Phase II (in the group of 362 examined twice, it refers to the most recent BMI). Type 2 diabetes and glucose intolerance (GINT) were defined by the National Diabetes Data Group criteria [6]. Classification of Type 2 diabetes by these criteria overlaps the WHO criteria while affording better discrimination of abnormal tolerance [23]. GINT comprised the nondiagnostic (which we termed borderline) and impaired tolerance categories as well as Type 2 diabetes. Hyperinsulinaemia was defined as the sum of the insulin levels at 60 and $120 \mathrm{~min}$ after glucose load (sum insulin) being above the 75 th percentile of sum insulin in the subgroup of nonobese normotensive persons with normal glucose tolerance (sum insu- 
Table 3. Prevalence (\%) of glucose intolerance and Type 2 diabetes by concurrent and past BMI category in the total study group

\begin{tabular}{|c|c|c|c|c|c|c|}
\hline & \multicolumn{3}{|c|}{$\begin{array}{l}\text { Glucose intolerance } \\
\text { Past BMI category }\end{array}$} & \multicolumn{3}{|c|}{$\begin{array}{l}\text { Type } 2 \text { diabetes } \\
\text { Past BMI category }\end{array}$} \\
\hline & $\begin{array}{l}\text { Higher than } \\
\text { concurrent }\end{array}$ & No change & $\begin{array}{l}\text { Lower than } \\
\text { concurrent }\end{array}$ & $\begin{array}{l}\text { Higher than } \\
\text { concurrent }\end{array}$ & No change & $\begin{array}{l}\text { Lower than } \\
\text { concurrent }\end{array}$ \\
\hline \multicolumn{7}{|l|}{ Concurrent BMI: } \\
\hline Low $(<23)$ & 30.4 & 22.4 & $\mathrm{X}$ & 6.5 & 3.1 & $\mathrm{X}$ \\
\hline Medium $(23-26.9)$ & 40.0 & 27.4 & 21.2 & 14.4 & 5.5 & 1.5 \\
\hline
\end{tabular}

$\mathrm{X}$ - Nonexistent, by definition

Table 4. Rate ratios for glucose intolerance and Type 2 diabetes comparing individuals who changed BMI categories over the 10-year follow-up period with those who did not change BMI category, including and excluding hypertensive patients

\begin{tabular}{|c|c|c|c|}
\hline BMI comparison groups: & \multicolumn{2}{|c|}{ Glucose intolerance } & $\begin{array}{l}\text { Type } 2 \text { diabetes } \\
\text { Total group } \\
n=2140\end{array}$ \\
\hline \multicolumn{4}{|l|}{ Past BMI higher than concurrent versus: } \\
\hline No-change individuals in same concurrent BMI category & $1.41(1.16-1.72)^{\mathrm{a}}$ & $1.54(1.04-2.29)^{\mathrm{a}}$ & $2.46(1.58-3.81)^{\mathrm{a}}$ \\
\hline No-change individuals in same Past BMI category & $0.93(0.69-1.25)^{d}$ & $0.98(0.66-1.43)^{d}$ & $1.30(0.86-1.98)^{\circ}$ \\
\hline $\begin{array}{l}\text { No-change individuals in same Past BMI category } \\
\text { Past BMI low } \\
\text { Past BMI medium }\end{array}$ & $\begin{array}{l}0.94(0.73-1.21)^{\mathrm{d}} \\
1.46(1.24-1.73)^{\mathrm{a}}\end{array}$ & $\begin{array}{l}0.99(0.72-1.36)^{\mathrm{d}} \\
1.67(1.31-2.13)^{\mathrm{a}}\end{array}$ & $\begin{array}{l}0.48(0.19-1.23)^{\mathrm{d}} \\
1.05(0.64-1.78)^{\mathrm{d}}\end{array}$ \\
\hline $\begin{array}{l}\text { Pattern summary - GINT } \\
\text { Ever } \geq 27 \text { versus always }<27 \\
\text { Always }<27 \text { : past }>23 \text { versus past }<23\end{array}$ & $\begin{array}{l}1.76(1.56-1.99)^{\mathrm{a}} \\
1.27(1.05-1.53)^{\mathrm{b}}\end{array}$ & $\begin{array}{l}1.86(1.56-2.21)^{\mathrm{a}} \\
1.19(0.94-1.52)^{\mathrm{b}}\end{array}$ & \\
\hline
\end{tabular}

${ }^{\mathrm{a}} p<0.01 ;{ }^{\mathrm{b}} p=0.02-0.05 ;{ }^{\mathrm{c}} p=0.12 ;{ }^{\mathrm{d}}$ not significant

Table 5. Effect of past and concurrent BMI category and changes in BMI units on rate of glucose intolerance (GINT) in the total study group a)

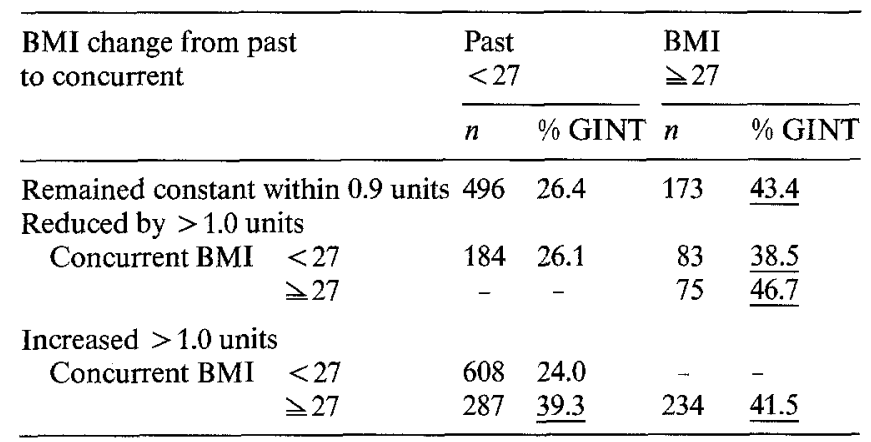

Rates among those $\supseteq 27$ at either examination are underlined

lin > PN75) [24]. Hypertension was defined either by reported use of antihypertensive medication or by at least two of the four blood pressure measurements exceeding $145 \mathrm{mmHg}$ systolic or $93 \mathrm{~mm}$ diastolic [24].

The independent effects of past and concurrent BMI on the rates of (1) GINT, (2) Type 2 diabetes and (3) hyperinsulinaemia in the total study group were analyzed in two ways:
1. BMI, both past and concurrent, was divided into three categories: low, <23; medium, 23-26.9; and high, $\geq 27$. Persons who changed BMI category over the follow-up period were compared with those whose category did not change, first within their past and again within their concurrent BMI category respectively, by Mantel-Haenszel rate ratios with $90 \%$ test-based confidence limits (equivalent to a onetailed 0.05 probability customarily used in this type of analysis) [26] as given in brackets following the rate ratios in the text and tables.

2. Logistic regression analysis (Biomedical Computer Programs, University of California, Program LR) was performed, with rate of GINT, Type 2 diabetes or hyperinsulinaemia respectively as the dependent variable. The independent variables were concurrent and past BMI as categorical variables, first as three categories (high, medium and low), then again as two categories (grouping together the medium and low ones, since their effects were not found to differ significantly), controlling for the possible confounding effects of age, sex and ethnic group. Results of the latter analysis were expressed as adjusted rate ratios, with $90 \%$ confidence limits derived from the logistic coefficients.

In the group of 362 individuals whose glucose tolerance was examined twice, analysis related the effect of past and concurrent BMI on the rate of individuals whose glucose tolerance category deteriorated, i. e. normal tolerance changing to GINT as well as borderline or impaired tolerance changing to Type 2 diabetes. This rate was the dependent variable in the logistic regression analysis, with past BMI and difference between past and concurrent BMI as continuous indepen- 
dent variables, again controlling for age, sex and ethnic group. The modified approach to analysis was adopted because of the smaller number of cases in this group. Results were expressed as risk of deterioration associated with increase of $1 \mathrm{BMI}$ unit.

Differences in variability, expressed as standard deviation, of sum insulin between BMI categories, were analysed by $\mathrm{F}$ test.

\section{Results}

\section{General}

Glucose intolerance (GINT) was found in $696(32.5 \%)$ and Type 2 (non-insulin-dependent) diabetes in 132 $(6.2 \%)$ of the 2140 participants.

The basic relationship of the rates of GINT and of Type 2 diabetes with obesity was demonstrated in 825 individuals with both past and concurrent BMI in the same two-unit interval (Fig. 1). Both rates rose gradually but non-linearly as BMI increased, with inflection points at $\mathrm{BMI} \geqslant 27$ for GINT and at $\mathrm{BMI} \supseteq 31$ for Type 2 diabetes.

With BMI grouped into three categories (low, medium and high), past and concurrent BMI categories were the same in $1392(65 \%)$ of the 2140 participants (nochange group), while $182(8.5 \%)$ had been in a higher category and $566(26.4 \%)$ in a lower category in the past (Table 1). In $94 \%$ of the latter 748 individuals BMI changed by at least one full unit; the majority shifted to the adjacent BMI category, with changes ranging from 2.6 to $9.7 \mathrm{~kg}$. Only $4.7 \%$ changed by two categories. It should be noted that these categories were chosen after preliminary analyses, based on greater numbers of narrower categories which yielded practically the same results as those described below.

In the no-change group, the GINT rate ratio for the medium BMI category relative to the low (27.4 versus 22.4 ) was 1.22 (1.01-1.48), rising to $1.72(1.50-1.97)$ for the high relative to the medium category ( 47.0 versus 27.4) (Table 2). This greater increase in rate from the medium to the high category persisted in all strata by sex, age, blood pressure and ethnic groups (except in Yemenites, in whom it rose almost twofold even in the medium compared with the low category $(p=0.001)$ with a similar increment from the medium to the high category), despite differences in absolute rates between strata (Table 2). Rates increased with age in all BMI categories $(p<0.0001)$. They were significantly lower in women then in men in the low and medium categories $(p<0.0001)$. Differences between ethnic groups were not significant in the low BMI category. However, in the medium and high categories the rates in Yemenites were significantly higher than in the other three groups $(p=0.0001)$. The same effect of BMI was present in normotensive subjects as well as in both untreated and treated hypertensive subjects, although the rate of GINT was significantly higher in both hypertensive categories $(p<0.001)$. Trends were similar for Type 2 diabetes alone.
Table 6. Rate of deterioration of glucose tolerance in 362 individuals with two OGTTs 3.5 years apart, by initial tolerance category, past and concurrent $\mathrm{BMI}^{\mathrm{a}}$

\begin{tabular}{lll}
\hline & $\begin{array}{l}\text { No. of } \\
\text { cases }\end{array}$ & $\begin{array}{l}\text { Rate }(\%) \text { of } \\
\text { deterioration }\end{array}$ \\
\hline Total group & 362 & 11.6 \\
Initial tolerance - normal & 279 & $11.8^{\mathrm{b}}$ \\
Initial tolerance - borderline & 83 & $10.8^{\mathrm{c}}$ \\
or impaired & & \\
Past and concurrent BMI $<27$ & 229 & 9.6 \\
Past BMI $\geq 27$ and concurrent $<27$ & 16 & 12.5 \\
Past BMI <27 and concurrent $\geq 27$ & 50 & 14.0 \\
Past and concurrent BMI $\geq 27$ & 67 & 16.4 \\
Rate ratio of deterioration in those ever & 1.56 & \\
$\geqslant 27$ versus those always $<27$ & $(0.97-2.52)$ & \\
\hline
\end{tabular}

a Past BMI refers to phase I BMI; Concurrent BMI refers to that at the 2nd OGTT

${ }^{b}$ Comprises 32 cases of change to borderline or impaired tolerance and one to diabetes

- Comprises 9 cases who changed to diabetes. Change from boderline to impaired tolerance was not defined as deterioration in this analysis

Table 7. Adjusted rate ratios and significance levels by logistic regression analysis of the net effect of past and concurrent BMI $\geq 27$ after accounting for age, sex and ethnic group

\begin{tabular}{|c|c|c|c|}
\hline \multirow[t]{2}{*}{$\begin{array}{l}\text { Analysed } \\
\text { group }\end{array}$} & \multirow{2}{*}{$\begin{array}{l}\text { Dependent } \\
\text { variable } \\
\text { rate of: }\end{array}$} & \multicolumn{2}{|l|}{$\begin{array}{l}\text { Rate ratios and } \\
\text { Significance levels }\end{array}$} \\
\hline & & Past BMI & Concurrent BMI \\
\hline $\begin{array}{l}\text { Total study } \\
\text { group } \\
(n=2140)\end{array}$ & $\begin{array}{l}\text { GINT } \\
\text { Type } 2 \\
\text { diabetes }\end{array}$ & $1.38(1.12-1.71)^{*}$ & $2.02(1.66-2.47)^{* *}$ \\
\hline $\begin{array}{l}\text { Two OGTTs } \\
(n=362)\end{array}$ & $\begin{array}{l}\text { Deterioration } \\
\text { of glucose } \\
\text { tolerance }\end{array}$ & $1.13(1.05-1.22)^{* *}$ & $1.05(0.93-1.18)$ \\
\hline $\begin{array}{l}\text { Insulin } \\
\text { response group } \\
(n=1200)\end{array}$ & $\begin{array}{l}\text { Hyper- } \\
\text { insulinaemia }\end{array}$ & $1.19(0.89-1.58)-$ & $2.61(2.02-3.37)^{* *}$ \\
\hline
\end{tabular}

a The lack of significance of the effect of concurrent BMI may be due to an insufficient number of cases $(n=50)$ whose past and concurrent BMI were $<27$ and $\geq 27$ respectively, in whom the effect of concurrent $\mathrm{BMI}$ is expected to be evident according to the cross-sectional data. BMI at the time of the 1st OGTT also had no effect on the deterioration.

b $* *<0.001 ; *<0.01 ;-$ N.S.

The independent effects of past and concurrent BMI on the rates of GINT and of Type 2 diabetes were evaluated by comparing individuals in past higher and past lower BMI categories, respectively, with the nochange group (see rates in Table 3 ; rate ratios, confidence limits and significance levels in Table 4) as follows:

\section{Effect of past and concurrent BMI category on GINT}

Individuals with higher past BMI. These had significantly higher rates of GINT than the no-change individuals with the same concurrent BMI (30.4 versus 22.4 , 

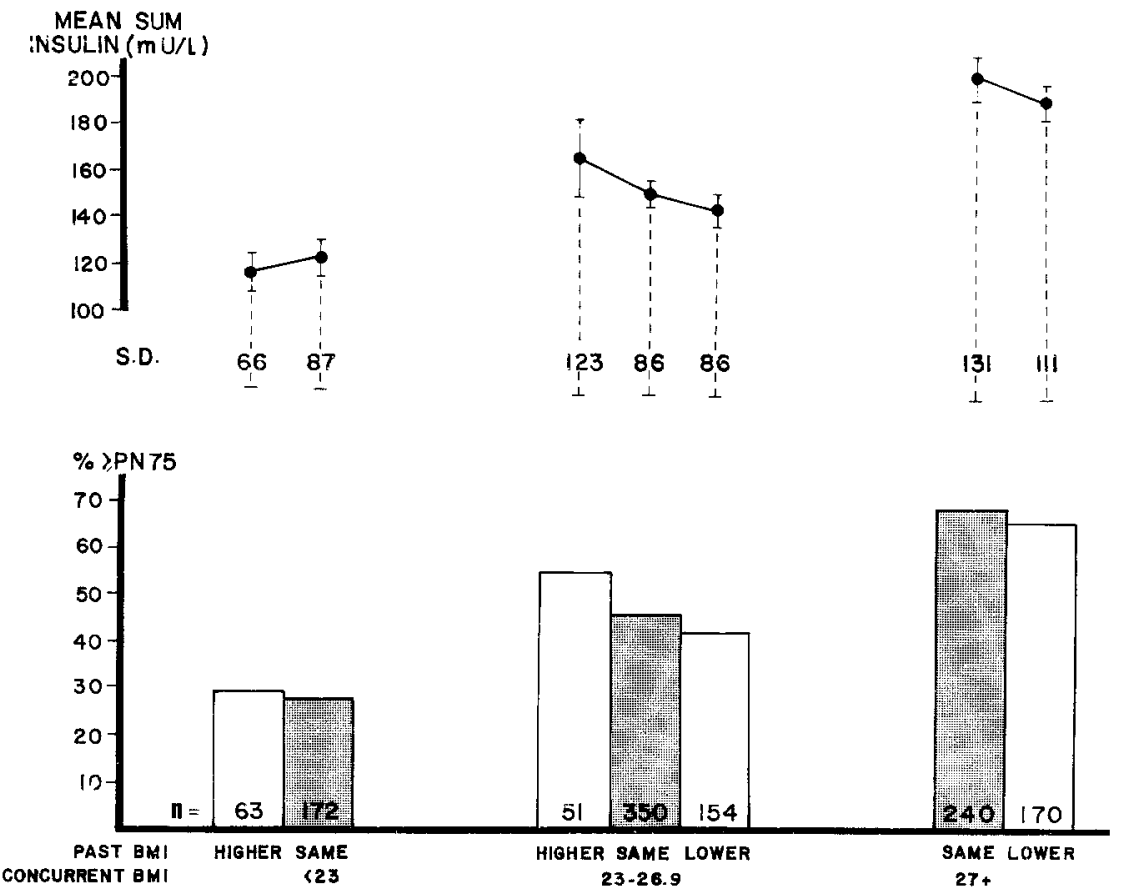

Fig. 2. Rate ( $\%$ ) of sum insulin $>$ PN75 and mean, standard deviation and standard error of sum insulin by concurrent and past body mass index (BMI). PN75 is the 75th percentile of sum insulin in normal-weight, normotensive subjects with normal glucose tolerance. Solid bracketed lines around the means represent standard errors. Broken bracketed lines represent standard deviations. Mean sum insulin and its rate $>$ PN75 increased significantly with concurrent $(p<0.001)$ but not past BMI. Within concurrent BMI categories standard deviations of sum insulin were significantly greater $(p<$ 0.05 ) when past $\mathrm{BMI}$ was $\geq 27$. Also, significantly higher standard deviations $(p<0.01)$ characterized the groups with BMI over $\geq 27$ versus those always $<27$
40.0 versus 27.4). Moreover, they resembled the nochange individuals with whom they had shared a BMI category in the past ( 30.4 versus $27.4,40.0$ versus 47.0 ). Thus, the rate of GINT in individuals who reduced their weight was determined primarily by their past BMI category.

Individuals with lower past BMI. These had mildly but significantly lower rates than the no-change individuals in the same concurrent BMI category (21.2 versus 27.4, 40.1 versus 47.0). When their concurrent BMI was medium (i.e. past BMI low), they resembled the nochange individuals in the same past BMI category (21.2 versus 22.4). However, when their concurrent BMI was high (i.e. past BMI medium) the rate was significantly higher than that in no-change individuals in the same past BMI category (40.1 versus 27.4). Thus, in individuals with concurrent high BMI category, the main determinant of the rate of GINT was concurrent BMI.

Pattern summary. In all, individuals with $\mathrm{BMI} \supseteq 27$ (whether past, concurrent or both) were characterized by significantly increased prevalence of GINT (40.0, $47.0,40.1)$ with a rate ratio of $1.76(1.56-1.99)$ relative to those always $<27$. Among the latter, the rate of GINT was unaffected by current BMI, and was mildly but significantly increased $(p<0.05)$ in individuals with past medium BMI (30.4 and 27.4) versus those with past low BMI (22.4 and 21.2). The same analysis, repeated for normotensive subjects alone, yielded similar effects of past and concurrent BMI on GINT as in the total group (Table 4).

\section{Effect of past and concurrent BMI category on Type 2 diabetes.}

Individuals with lower or higher past BMI. Rates of Type 2 diabetes among all individuals who changed
BMI category differed significantly from those in nochange individuals in the same concurrent BMI category (Tables 3 and 4). The rate was higher among those whose past BMI was higher (6.5 versus 3.1 and 14.4 versus 5.5), and lower in those whose past BMI was lower (1.5 versus 5.5 and 5.7 versus 10.6$)$. Rates of Type 2 diabetes among those who changed category did not differ significantly from the rates among the no-change individuals in the BMI category to which they had belonged in the past ( 6.5 versus $5.5,14.4$ versus $10.6 ; 1.5$ versus 3.1 and 5.7 versus 5.5 respectively).

Pattern summary. The rate of Type 2 diabetes was determined in all individuals by past BMI, with no apparent effect of concurrent BMI. A twofold increase in this rate was associated with past high versus past medium BMI as well as with past medium versus past low BMI. It should be noted that of the 132 diabetic patients. Nineteen reduced while 21 increased their BMI category, the majority remaining unchanged.

\section{Effect of unit change in BMI: Dependence on whether maximum $B M I \geqslant 27$}

Weight changes in themselves, expressed in BMI units rather than categories, had no effect on the rate of GINT (Table 5) unless leading to BMI $\geq 27$. Thus the similarly low rates in individuals with both past and concurrent $\mathrm{BMI}<27$ as well as the similarly high rates in those whose BMI was ever $\geqslant 27$ regardless of whether BMI had decreased, increased or stayed the same, reemphasize that the main determinant of the rate of GINT was $\mathrm{BMI} \supseteq 27$ at either examination. The same analysis for the rate of Type 2 diabetes showed again that it was determined only by past BMI with no effect of subsequent weight changes. 


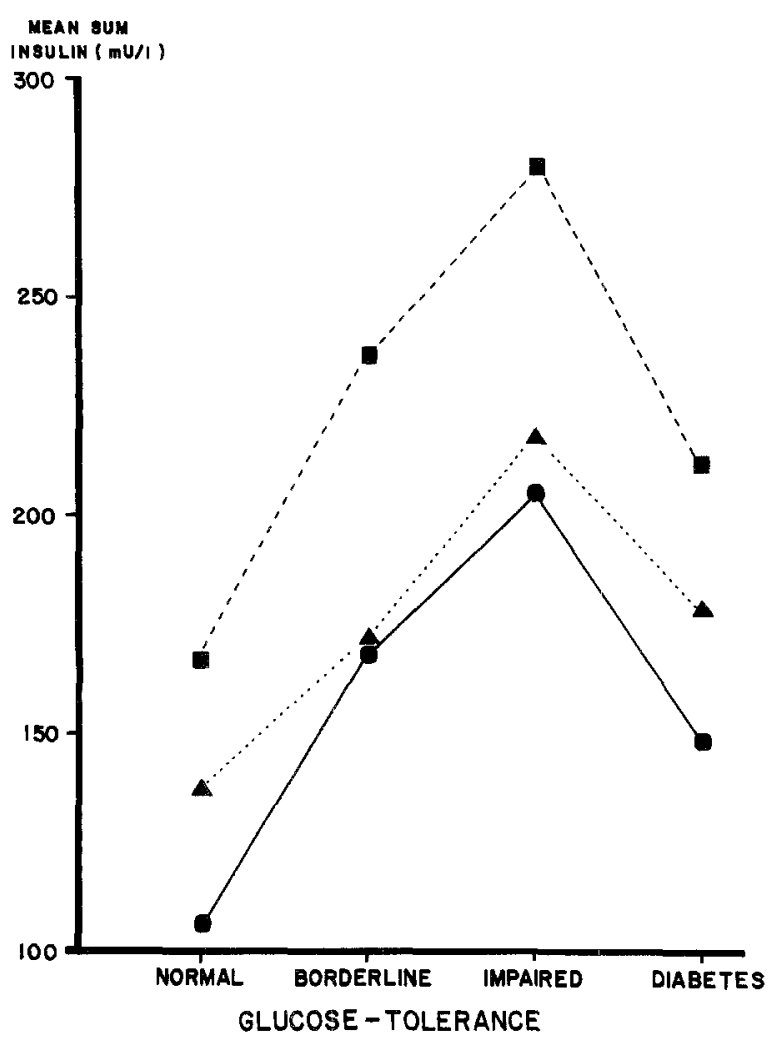

Fig.3. Mean sum insulin by glucose tolerance and concurrent body mass index (BMI) category $(\longrightarrow<23 ; \boldsymbol{\Lambda} \longrightarrow \ldots$ 23-26.9; - $\geq 27)$. Mean sum insulin increased significantly $(p<0.001)$ from normal through borderline to impaired glucose tolerance and decreased significantly $(p<0.001)$ in diabetes relative to impaired tolerance. Response was similar in the two lower categories and significantly higher $(p<0.001)$ in $\mathrm{BMI} \supseteq 27$

\section{Effect of past and concurrent BMI on deterioration of glucose tolerance}

In the 362 individuals who had two OGTTs, the overall rate of deterioration of glucose tolerance was $11.6 \%$ and similar in magnitude whether initially normal, borderline or impaired (Table 6). Here too, the same threshold effect of $\mathrm{BMI} \geq 27$ was found (Table 6). The rate ratio for deterioration among those with $\mathrm{BMI} \geq 27$ at any point to deterioration in those with BMI always $<27$ was $1.56(0.97-2.52)$, similar to the parallel cross-sectional rate ratio of 1.76 (Table 4) for GINT in the total study group. Although this rate ratio was only on the border of significance, past BMI was significantly associated with the rate of deterioration in the logistic regression analysis $(p<0.01$, Table 7$)$.

\section{Effect of past and concurrent BMI on insulin response}

In individuals who did not change BMI category, insulin response increased with increasing BMI as expected $(p<0.001)$ from 123 in the low through 150 in the medium and 190 in the high BMI category. In individuals who changed BMI category, insulin response was similar to that of no-change individuals in the same concurrent BMI category (Fig.2). Within concurrent BMI categories the variability of sum insulin as expressed by the respective standard deviations was significantly greater $(p<0.05)$ in individuals whose past BMI was $\geq 27$ (123 versus 86 and 131 versus 111). Moreover, highly significant excess variability characterized all three groups with $\mathrm{BMI}$ ever $\geq 27$, as compared with those always $<27(123,131$ and 111 versus $66,87,86$ and 86$)$. This excess variability resided completely in the glucose-intolerant categories, as the respective standard deviations for persons with normal tolerance within these three groups were 65, 97 and 85 (not presented in Figures). Within glucose tolerance categories the significant $(p<0.001)$ effect of concurrent BMI on sum insulin was mainly associated with $\mathrm{BMI} \geq 27$ while levels were similar in the two lower BMI categories (Fig. 3). Insulin response increased significantly $(p<0.001)$ in all three concurrent BMI categories from normal through borderline to impaired tolerance, and decreased significantly $(p<0.001)$ in diabetes relative to impaired tolerance although remaining above normal.

\section{Multivariate analysis}

All effects of past and concurrent BMI were verified by logistic regression analysis, controlling for age, sex and ethnic group (Table 7).

\section{Discussion}

The universal association of concurrent obesity and GINT has been amply demonstrated in cross-sectional studies in diverse populations [1-6]. Longitudinal studies of samples of free-living general populations as well as of individuals with impaired glucose tolerance support an aetiological role for obesity in subsequent development of Type 2 diabetes [17-20, 27-30]. The effect is believed to be due to the hyperinsulinaemia and insulin resistance associated with obesity $[12-14]$. Accordingly a vicious circle is initiated. The hyperinsulinaemia causes down-regulation of insulin receptors in target tissues, further increasing insulin resistance and leading with time to impaired glucose tolerance, eventual B cell exhaustion and overt diabetes. This prevailing hypothesis assigns an essential role to the time element in the association of obesity and GINT. Indeed, overweight in the distant past was shown to be more determinative of the rate of worsening of impaired glucose tolerance to diabetes than overweight in the immediate past [27-30], but the relative roles of past and concurrent overweight have not been quantitatively evaluated. With respect to deterioration of normal to impaired glucose tolerance, only indirect evidence is available [31, 32]. A potential confounding factor ignored in all these studies is the effect of antihypertensive medications, which cause deterioration of glucose tolerance [21, 22] and are important here in view of the increased prevalence of hypertension among the obese $[33,34]$.

Our analysis was based on a representative population sample, accounted for the potential confounding 
effect of antihypertensive medications, and related quantitatively to the effect of both past and concurrent degree of overweight on the full range of GINT. It showed that:

a) The main determinant of the rate of Type 2 diabetes was the degree of obesity ten years earlier, while concurrent degree of overweight and interim weight changes had little effect. It is interesting that the majority of our Type 2 diabetic patients (all newly-found and asymptomatic) did not change BMI category, while the small number of those who lost weight was similar to that of those who gained weight. Since, as in the non-diabeticsubject, other reasons for weight loss must also have been present, diabetes-related weight loss was not more than a marginal factor in our study group.

b) The rate of GINT was affected by past as well as concurrent $\mathrm{BMI} \geq 27$, both of which were associated with an almost twofold increase in rate. Apart from an increase over the threshold of BMI 27, weight changes in either direction had only a minor effect on the rate of GINT.

c) The association with degree of overweight was nonlinear, with threshold effects of BMI $\supseteq 27$ for GINT, and $\geq 31$ for Type 2 diabetes, which were evident in all age, sex and blood pressure categories and in three of our four ethnic groups.

Similar non-linearity and inflection points were found in other studies in a variety of populations [17, 18]. The only exception in our study was the Yemenite group, in which the increase in rate was linear, increasing steeply even at intermediate excess of weight. It is intriguing to speculate to what extent this sensitivity of Yemenites to the slightest degree of overweight plays a role in the transition from their reported zero rate of diabetes upon immigration to Israel [35] to their current exceedingly high rate.

d) Mean insulin response was associated only with concurrent BMI, again with a more pronounced effect of $\mathrm{BMI} \geq 27$. The effect of past BMI manifested itself in significantly greater variability of sum insulin. This finding, as well as the fact that all excess variability was associated with glucose intolerant individuals, is compatible with the vicious circle hypothesis, which predicts two opposing effects. Past high BMI is expected to be associated with increasingly high insulin levels. Conversely, eventual islet B-cell exhaustion, which should be most evident in this group, is expected to manifest itself as reduced insulin response [12-14]. As Type 2 diabetes with its attendant reduced insulin level, as well as impaired glucose tolerance with its exceedingly high insulin response, were both most prevalent in our high past BMI group, it seems that both these opposing effects were present and to some extent cancelled each other out, masking the effect of past BMI on mean insulin response.

The potential effect on our results of selective mortality between Phase I and Phase II must be addressed. As in other studies $[34,36]$, mortality among Phase I participants was higher in the high BMI category (Modan et al, unpublished data). While glucose tolerance was not examined in Phase I, in all likelihood Phase I diabetic patients, and perhaps persons with impaired glucose tolerance as well, would here have a higher mortality rate. This would tend to decrease the apparent effect of past BMI, and thus does not invalidate our results.

In conclusion, our findings show that past degree of overweight determines the rates of both Type 2 diabetes and impaired glucose tolerance ten years later. It seems reasonable to assume that high $\mathrm{BMI}$ in the more distant past will also have a diabetogenic effect. A concurrent high degree of overweight affects the rate of impaired glucose tolerance but not of Type 2 diabetes. Thus deterioration of normal to impaired glucose tolerance seems to occur shortly after BMI increases $\geq 27$. The effect of moderately high BMI is apparently more protracted. For the development of Type 2 diabetes with its associated reduced insulin response, a prolonged period of obesity seems to be required. These findings are compatible with the "late diabetogenic effect" suggested by Keen at al [27], as well as with the vicious circle hypothesis described above. The threshold effect of $\mathrm{BMI} \supseteq 27$, both on rate of GINT and on insulin response, suggests a "critical mass" of fat tissue which accelerates deterioration of glucose tolerance. While improvement in glucose tolerance and insulin sensitivity accompanies weight reduction in short term studies, our data seem to indicate that in the long term reversibility of the process is limited, at least in the range of weight loss which characterized our study group.

Acknowledgements. The study was funded by the US National Institute of Arthritis, Diabetes, Digestive and Kidney Diseases, by the German-Israeli Fund and by the Binational US-Israel Fund.

\section{References}

1. Joslin EP (1921) The prevention of diabetes mellitus. JAMA 76: 79-84.

2. John HF (1929) A summary of findings in 1100 glucose tolerance estimations. Endocrinology 13: 388-392

3. West KM (1978) Epidemiology of diabetes and its vascular lesions. Elsevier - North Holland, New York

4. WHO Expert Committee on Diabetes Mellitus (1980) Technical Report Series No.646. Geneva: World Health Organization

5. Zimmet P (1982) Type 2 (non-insulin-dependent) diabetes - an epidemiologic overview. Diabetologia 22: 399-411

6. National Diabetes Data Group (1979) Classification and diagnosis of diabetes mellitus and other categories of glucose intolerance. Diabetes 28: 1039-1057

7. Himsworth HP (1936) Diabetes mellitus: Its differentiation into insulin sensitive and insulin insensitive types. Lancet 1: 117-120 
8. Yalow RS, Berson SA (1960) Plasma insulin concentrations in non-diabetics and early diabetic subjects. Determinations by a new sensitive immunoassay technique. Diabetes 9: 254-260

9. Rabinowitz D, Zierler KL (1962) Forearm metabolism in obesity and its response to intra-arterial insulin: Characterization of insulin resistance and evidence for adaptive hyperinsulinism. J Clin Invest $41: 2173-2181$

10. Karam JH, Grodsky GM, Forsham PH (1963) Excessive insulin response to glucose in obese subjects as measured by immunochemical assay. Diabetes 12: 197-204

11. Abrams ME, Jarrett RJ, Keen H, Boyns DR, Crossley JH (1969) Early glucose tolerance and related factors in a normal population sample. II. Interrelationship of glycerides, cholesterol and other factors with glucose and insulin response. Br Med J 1: 599-602

12. Olefsky JM, Kolterman OJ, Scarlett JA (1982) Insulin action and resistance in obesity and non-insulin dependent type II diabetes mellitus. Am J Physiol 243: E 15-30

13. DeFronzo RA, Ferrannini E (1982) The pathogenesis of non-insulin-dependent diabetes: An update. Medicine 61:125-140

14. Reaven GM (1980) Insulin independent diabetes mellitus. Metabolic characteristics. Metabolism 29: 445-454

15. Halverson JD, Kramer J, Cave A, Permutt A, Santiago J (1982) Altered glucose tolerance, insulin response and insulin sensitivity after massive weight reduction subsequent to gastric bypass. Surgery $92: 235-240$

16. Grunberger G, Taylor SI, Dons RF, Gorden P (1983) Insulin receptors in normal and disease states. Clin Endocrinol Metabol 12: 191-219

17. Westlund K, Nicolaysen R (1972) Ten-year mortality and morbidity related to serum cholesterol. Scan J Clin Lab Invest 30 [Supp] 127]: $1-24$

18. Knowler WC, Pettitt DJ, Savage PJ, Bennett PH (1981) Diabetes incidence in Pima Indians: contributions of obesity and parental diabetes. Am J Epidemiol 113: 144-156

19. Medalie JH, Papier CM, Goldbourt U, Herman JB (1975) Major factors in the development of diabetes mellitus in 10000 men. Arch Intern Med 135: 811-817

20. Butler WJ, Ostrander LD Jr., Carmen WJ, Lamphiear DE (1982) Diabetes mellitus in Tecumseh, Michigan. Prevlance, incidence and associated conditions. Am J Epidemiol 116: 971-980

21. Murphy MB, Kohner E, Lewis PJ, Schumer B, Dollery CT (1982) Glucose intolerance in hypertensive patients treated with diuretics; a fourteen-year follow-up. Lancet 2: 1293-1295

22. Flamenbaum W (1983) Metabolic consequences of antihypertensive therapy. Annals of Intern Med 98: 875-880

23. Modan M, Halkin H, Karasik A, Lusky A (1984) Effectiveness of glycosylated hemoglobin, fasting plasma glucose, and a single post load plasma glucose level in population screening for glucose intolerance. The Israel Study of Glucose Intolerance, Obesity and Hypertension. Am J Epidemiol 119: 431-444

24. Modan M, Halkin H, Almog S, Lusky A, Eshkol A, Shefi M, Shitrit A, Fuchs Z (1985) Hyperinsulinemia - a link between hypertension obesity and glucose intolerance. J Clin Invest 75: 809-817

25. Cerasi E, Efendic S, Luft R (1973) Dose-response relation between plasma-insulin and blood-glucose levels during oral glucose loads in prediabetic and diabetic subjects. Lancet 1:794-797

26. Miettinen OS (1976) Estimability and estimation in case referent studies. Am J Epidemiol 103: 226-235

27. Keen H, Jarrett RJ, McCartney P (1982) The ten-year follow-up of the Bedford survey (1962-1972): glucose tolerance and diabetes. Diabetologia 22: 73-78

28. King H, Zimmet P, Raper LR, Balkau B (1984) The natural history of impaired glucose tolerance in the Micronesian population of Nauru: A six-year follow-up study. Diabetologia 26:39-43

29. Sasaki A, Suzuki T, Horiuchi N (1982) Development of diabetes in Japanese subjects with impaired glucose tolerance: A seven-year follow-up study. Diabetologia 22: 154-157

30. Kadowaki T, Miyake Y, Hagura R, Akanuma Y, Kajinuma $\mathrm{H}, \mathrm{Ku}-$ zuya N, Takaku F, Kosaka K (1984) Risk factors for worsening to diabetes in subjects with impaired glucose tolerance. Diabetologia 26: $44-49$

31. Noppa H (1980) Body weight change in relation to incidence of ischemic heart disease and change in risk factors for ischemic heart disease. Am J Epidemiol 111: 693-704

32. Ashley FW Jr., Kannel WB (1974) Relation of weight change to changes in atherogenic traits: the Framingham study. J Chron Dis 27: $103-114$

33. Chiang BN, Perlman LV, Epstein FH (1969) Overweight and hypertension. A review. Circulation 39: 403-421

34. Sims EAH (1982) Mechanisms of hypertension in the overweight. Hypertension 4 (Suppl III): III-43-III-49

35. Cohen AM (1961) Prevalence of diabetes among different ethnic Jewish groups in Israel. Metabolism 10:50-58

36. Keys A (1980) W.O.Atwater Memorial Lecture: Overweight, obesity, coronary heart disease and mortality. Nutr Rev 38: 297-307

Received: 17 April 1985

and in revised form: 26 September 1985

Michaela Modan

Head, Biometry Unit

Department of Clinical Epidemiology

Chaim Sheba Medical Center

Tel Hashomer 52621, Istael 